\title{
Inducing the Einstein action in QCD-like theories
}

\author{
John F. Donoghue* \\ Department of Physics, University of Massachusetts Amherst, Massachusetts 01003, USA \\ Gabriel Menezes ${ }^{\dagger}$ \\ Department of Physics, University of Massachusetts Amherst, Massachusetts 01003, USA \\ and Departamento de Física, Universidade Federal Rural do Rio de Janeiro, \\ 23897-000 Seropédica, Rio de Janeiro, Brazil
}

(Received 16 January 2018; published 27 March 2018)

\begin{abstract}
We evaluate the induced value of Newton's constant which would arise in QCD. The ingredients are modern lattice results, perturbation theory and the operator product expansion. The resulting shift in the Planck mass is positive. A scaled-up version of such a theory may be part of a quantum field theory treatment of gravity.
\end{abstract}

DOI: 10.1103/PhysRevD.97.056022

\section{INTRODUCTION}

The action for a pure Yang-Mills theory, such as the gluonic sector of QCD, is scale invariant. Nevertheless, a scale enters the theory through the running coupling constant, which defines an energy scale at which the coupling becomes large. The spectrum and observables of the theory will depend on this scale through dimensional transmutation.

In particular, when we include the metric as a field as we do in general relativity, QCD will induce a change in the cosmological constant and in the gravitational constant $G$. Since the cosmological constant is related to the energy density of the vacuum, the QCD contribution to it has a simple expression in terms of the trace of the energymomentum tensor

$$
4 \Lambda_{\text {ind }}=\left\langle 0\left|T_{\mu}^{\mu}\right| 0\right\rangle
$$

where $|0\rangle$ is the vacuum. The shift in the gravitational constant is given by the Adler-Zee formula [1-5] (to be reviewed in Sec. II) in terms of the correlation function

$$
\frac{1}{16 \pi G_{\text {ind }}}=\frac{i}{96} \int d^{4} x x^{2}\langle 0| T\left(T_{\mu}^{\mu}(x) T_{\nu}^{\nu}(0)|0\rangle\right.
$$

\footnotetext{
*donoghue@physics.umass.edu

†gsantosmenez@umass.edu
}

Published by the American Physical Society under the terms of the Creative Commons Attribution 4.0 International license. Further distribution of this work must maintain attribution to the author(s) and the published article's title, journal citation, and DOI. Funded by SCOAP ${ }^{3}$.
In this paper we provide a determination of the induced gravitational constant defined by the Adler-Zee formula in QCD.

In gluonic QCD, the trace of the energy-momentum tensor is given by the trace anomaly

$$
T_{\mu}^{\mu}=\frac{\beta(g)}{2 g} F_{\mu \nu}^{a} F^{a \mu \nu}
$$

where $\beta(g)$ is the renormalization-group beta function of QCD and $g$ is the (dimensionless) coupling constant. The Adler-Zee formula involves the correlation function of $F^{2}$, which has been studied in the context of scalar glueballs. The ingredients needed for the evaluation are then perturbation theory [4] and the operator product expansion (OPE) [6-8] at short distances and modern lattice glueball studies [9] at long distances. We match these contributions at a distance/energy scale $x^{-1}=X_{0}^{-1}=2 \mathrm{GeV}$ (in units of $\hbar=c=k_{B}=1$, which will be consistently employed throughout the paper). While there is some residual matching dependence, this procedure determines that the induced $G$ is positive and evaluates its magnitude to within about $30 \%$.

While this calculation can be considered as simply a demonstration of a feature of QCD, there is potentially another motivation in gravitational physics. Strongly interacting theories similar to QCD could occur at higher energies also. There would be shifts in the gravitational constant also in such theories. Perhaps the effective Planck mass

$$
M_{P}^{2}=\frac{1}{G}
$$

is in fact determined by the strongly interacting theory with the largest intrinsic scale. This would occur if the 
coefficient of the Einstein action in the ultimate theory of quantum gravity was smaller than the Yang-Mills scale or absent altogether, as would happen in scale-/conformally invariant gravitational theories [10-13]. So perhaps the Planck scale is a manifestation of a high-scale Yang-Mills theory. The fact that the QCD result comes out to be positive is important for such a possibility. We do not analyze such gravitational theories in this paper, but we plan to return to that topic in future work.

The outline of the paper is as follows. In Sec. II we briefly review the origin of the Adler-Zee formula. In the subsequent section, we collect the various ingredients for the evaluation. Section IV is devoted to a numerical evaluation as well as a discussion of the uncertainties. In Sec. V we provide a summary. The Appendix is devoted to reconciling a (previously unnoticed) discrepancy in the literature involving a related sum-rule formula for the cosmological constant, where the works of Novikov et al. [6] and of Brown and Zee [14] yield sum rules that differ by a factor of 2 .

\section{BRIEF REVIEW OF THE ADLER-ZEE FORMULA}

Here we review the induced gravitational effects due to the matter sector of a quantum field theory coupled to the metric. We take the Minkowski metric as $\eta_{\mu \nu}=$ $\operatorname{diag}(1,-1,-1,-1)$ and the Riemann curvature tensor given by $R_{\mu \nu \kappa}^{\lambda}=\partial_{\kappa} \Gamma_{\mu \nu}^{\lambda}+\Gamma_{\mu \nu}^{\eta} \Gamma_{\kappa \eta}^{\lambda}-(\nu \leftrightarrow \kappa)$. We define the gravitational effective action by

$$
e^{i S_{\text {eff }}\left[g_{\mu \nu}\right]}=\int d \phi e^{i S_{\text {mater }}\left[\phi, g_{\mu \nu}\right]},
$$

where $\phi$ represents generically the matter fields and $S_{\text {matter }}\left[\phi, g_{\mu \nu}\right]$ describes matter fields on a curved background. The action $S_{\text {eff }}\left[g_{\mu \nu}\right]$ is a scalar under general coordinate transformations. This observation allows one to represent it as the integral over the manifold of a scalar density. Formally, for slowly varying metrics, one has the following series expansion in powers of $\partial_{\lambda} g_{\mu \nu}$ :

$$
\begin{aligned}
S_{\text {eff }}\left[g_{\mu \nu}\right] & =\int d^{4} x \sqrt{-g} \mathcal{L}_{\text {eff }}\left[g_{\mu \nu}\right], \\
\mathcal{L}_{\text {eff }}\left[g_{\mu \nu}\right] & =\mathcal{L}_{\text {eff }}^{(0)}\left[g_{\mu \nu}\right]+\mathcal{L}_{\text {eff }}^{(2)}\left[g_{\mu \nu}\right]+\mathcal{O}\left[\left(\partial_{\lambda} g_{\mu \nu}\right)^{4}\right], \\
\mathcal{L}_{\text {eff }}^{(0)}\left[g_{\mu \nu}\right] & =-\Lambda_{\text {ind }}, \quad \mathcal{L}_{\text {eff }}^{(2)}\left[g_{\mu \nu}\right]=\frac{R}{16 \pi G_{\text {ind }}} .
\end{aligned}
$$

Our task is to derive representations for the induced cosmological constant $\Lambda_{\text {ind }}$ and the induced Newton gravitational constant $G_{\text {ind }}$ in terms of the vacuum expectation value of products of the stress-energy tensor $T_{\mu \nu}$ of the matter fields. For a discussion on the conditions that $\Lambda_{\text {ind }}$ and $G_{\text {ind }}$ should satisfy in order to be uniquely calculable in terms of the renormalized parameters of the flat space-time matter theory, see the review [4]. Here we consider a matter Lagrangian - in our case QCD-coupled covariantly to the gravitational field. For weak fields the metric can be expanded using $g_{\mu \nu}=\eta_{\mu \nu}+h_{\mu \nu}$. One finds

$$
\begin{aligned}
\mathcal{L}_{\text {matter }}\left[g_{\mu \nu}, A_{\mu}\right]= & \mathcal{L}_{0}\left[\eta_{\mu \nu}, A_{\mu}\right]-\frac{1}{2} h^{\mu \nu} T_{\mu \nu} \\
& +\frac{1}{4} h^{\mu \nu} h^{\alpha \beta} \tau_{\mu \nu, \alpha \beta}+\cdots
\end{aligned}
$$

On the right-hand side of this equation all the indices are raised and lowered with the flat space metric. The term linear in $h^{\mu \nu}$ yields the energy-momentum tensor and there is a term quadratic in $h^{\mu \nu}$ also. The latter will be discussed in the Appendix as it plays a role in the cosmological constant sum rule.

The effective action for the gravitational field will then include contributions from the interactions of the matter fields. An expansion in powers of the field $h^{\mu \nu}$ yields

$$
\begin{aligned}
i S_{\mathrm{eff}}[h]= & -\frac{i}{2} \int d^{4} x h^{\mu \nu}(x)\left\langle T_{\mu \nu}(x)\right\rangle \\
& +\frac{i}{4} \int d^{4} x h^{\mu \nu}(x) h^{\alpha \beta}(x)\left\langle\tau_{\mu \nu, \alpha \beta}(x)\right\rangle \\
& +\frac{i^{2}}{2 !}\left(\frac{1}{2}\right)^{2} \int d^{4} x \int d^{4} y h^{\mu \nu}(x) h^{\rho \sigma}(y) \\
& \times\left\langle T\left\{\bar{T}_{\mu \nu}(x) \bar{T}_{\rho \sigma}(y)\right\}\right\rangle+\mathcal{O}\left(h^{3}\right)
\end{aligned}
$$

where $\langle\ldots\rangle=\langle 0|\ldots| 0\rangle$ denotes a vacuum expectation value and $\bar{T}^{\mu \nu}(x)=T^{\mu \nu}(x)-\left\langle T^{\mu \nu}(x)\right\rangle$. The above expression is very similar to the usual expansion for the generating functional of connected correlation functions in quantum field theory, as long as one envisages $h_{\mu \nu}(x)$ as an external field. As alluded to above, the extra contribution coming from the second term on the right-hand side is necessary for consistency.

Following Zee [2], at this stage it is most convenient (but not required [14]) to specialize to the trace of $h_{\mu \nu}$, using $h_{\mu \nu}(x)=\frac{1}{4} \eta_{\mu \nu} h(x)$. In this case the action only involves the trace of the energy-momentum tensor, $T(x)=\eta_{\mu \nu} T^{\mu \nu}(x)$. For long-wavelength metric fields, in our case wavelengths longer than the QCD scale, we can Taylor expand $h(y)$

$$
\begin{aligned}
h(y)= & h(x)+(y-x)^{\mu} \partial_{\mu} h(x) \\
& +\frac{1}{2}(y-x)^{\mu}(y-x)^{\nu} \partial_{\mu} \partial_{\nu} h(x)+\cdots
\end{aligned}
$$

The effective Lagrangian can then be identified as 


$$
\begin{aligned}
i \mathcal{L}_{\text {eff }}[h]= & -\frac{i}{2} h(x)\left[\frac{1}{4}\langle T(x)\rangle\right]+\frac{i}{16}(h(x))^{2}\left[\frac{1}{4} \eta^{\mu \nu} \eta^{\rho \sigma}\left\langle\tau_{\mu \nu, \rho \sigma}(x)\right\rangle\right]-\frac{1}{16}(h(x))^{2} \int d^{4} z\left[\frac{1}{8}\langle T\{\bar{T}(z) \bar{T}(0)\}\rangle\right] \\
& +\frac{1}{2^{10}}\left(\partial_{\mu} h\right)^{2} \int d^{4} z z^{2}[\langle T\{\bar{T}(z) \bar{T}(0)\}\rangle]+\mathcal{O}\left(h^{3}\right) .
\end{aligned}
$$

The terms without any derivatives of $h$ are related to the cosmological constant. In particular the term linear in $h$ reproduces Eq. (1). The Einstein action involves two derivatives of the metric. For the form of the metric used here we have

$$
\sqrt{-g} R=-\frac{3}{32}\left(\partial_{\mu} h(x)\right)^{2}+\cdots
$$

Comparing the induced Lagrangian with the EinsteinHilbert Lagrangian, one finds the Adler-Zee formula describing the QCD contribution to the Einstein-Hilbert action

$$
\frac{1}{16 \pi G_{\text {ind }}}=\frac{i}{96} \int d^{4} z z^{2}\langle T\{\bar{T}(z) \bar{T}(0)\}\rangle .
$$

Notice that this sum rule involves the vacuum-subtracted version of the energy-momentum tensor, and we will treat this as being implied in subsequent work.

The Adler-Zee formula involves the two-point function of the trace of the energy-momentum tensor, which (following Adler) we call $\Psi(x)$

$$
\Psi(x)=\langle T\{\bar{T}(x) \bar{T}(0)\}\rangle .
$$

The Fourier transform of this gives the momentum-space correlator

$$
\Pi_{A Z}\left(q^{2}\right)=-i \int d^{4} x e^{i q \cdot x}\langle T\{\bar{T}(x) \bar{T}(0)\}\rangle
$$

In terms of the correlator, the induced gravitational constant involves the first derivative at zero momentum

$$
\frac{1}{16 \pi G_{\text {ind }}}=\frac{1}{12} \Pi_{A Z}^{\prime}(0)
$$

In the course of evaluating the induced value of $G$, we will work in Euclidean space. The corresponding formulas there are

$$
\frac{1}{16 \pi G_{\text {ind }}}=-\frac{1}{96} \int d^{4} y_{E} y^{2}\langle T\{\bar{T}(y) \bar{T}(0)\}\rangle
$$

and

$$
\frac{1}{16 \pi G_{\text {ind }}}=-\left.\frac{1}{12} \frac{d}{d Q^{2}} \Pi_{A Z}\left(Q^{2}\right)\right|_{Q^{2}=0}
$$

\section{INGREDIENTS OF THE SUM RULE}

The integration in the Adler-Zee formula runs over all distances. At long distances we are unable to perform analytical calculations. However, this particular correlation function is related to one which has been used to determine glueball properties, and has been studied on the lattice. We will use the most recent lattice work which yields the parameters which we will need [9]. However the lattice studies do not probe the shorter-distance properties. At the shortest distance, the perturbative contributions have been calculated by Adler [4]. In the intermediate energy range, there are QCD sum-rule techniques, dating back to the work of Novikov et al. (NSVZ) [6], which use the OPE to describe some contributions which are subleading to the perturbative contribution but still relevant at moderate energies. We will separate the problem into the longand short-distance contributions. We tie them together at a distance which corresponds to an energy of $2 \mathrm{GeV}$.

After performing a change of variables $x^{2}=t$, we split the integration into an ultraviolet part and an infrared part as follows:

$$
\begin{aligned}
\frac{1}{16 \pi G_{\mathrm{ind}}} & =-\frac{\pi^{2}}{96}\left(I_{\mathrm{UV}}+I_{\mathrm{IR}}\right), \\
I_{\mathrm{UV}} & =\int_{0}^{t_{0}} d t t^{2} \Psi(t), \\
I_{\mathrm{IR}} & =\int_{t_{0}}^{\infty} d t t^{2} \Psi(t) .
\end{aligned}
$$

The high-energy portion $I_{\mathrm{UV}}$ contains perturbative contributions coming from short-distance scales as well as terms coming from intermediate energies which will be assessed through an operator product expansion technique as mentioned above.

As discussed previously, the infrared part $I_{\mathrm{IR}}$ will be estimated within lattice methods. For large Euclidean $x$, one considers that $\Psi_{\mathrm{IR}}$ (the infrared part of $\Psi$ ) takes the form of the correlation function for a massive scalar particle:

$\Psi_{\mathrm{IR}}(x)=\frac{\lambda^{2}}{4 \pi^{2}} \frac{M_{g}}{x} K_{1}\left(M_{g} x\right)=\lambda^{2} \int \frac{d^{4} p}{(2 \pi)^{4}} \frac{e^{i \mathbf{p} \cdot \mathbf{x}+i p_{4} \tau}}{\mathbf{p}^{2}+p_{4}^{2}+M_{g}^{2}}$,

where $K_{1}(z)$ is a modified Bessel function, $x=\sqrt{|\mathbf{x}|^{2}+\tau^{2}}$ ( $\tau$ is the "Euclidean time"), $M_{g}$ is the glueball mass and 


$$
\lambda=\langle 0|\bar{T}(0)| S\rangle,
$$

is the glueball coupling, with $|S\rangle$ being the normalized scalar glueball state.

Let us discuss the perturbative contribution accommodated by the high-energy/short-distance component, $I_{\mathrm{UV}}$. It can be calculated directly in position space and its form is given by [4]

$$
\Psi_{\text {pert }}=\frac{C_{\Psi}}{x^{8}\left(\log \left(1 / \Lambda_{\mathrm{QCD}}^{2} x^{2}\right)\right)^{2}}, \quad C_{\Psi}=\frac{96}{\pi^{4}}
$$

where $\Lambda_{\mathrm{QCD}}$ is the QCD scale parameter. We note the highly singular nature of the correlator at short distance, especially the $x^{8}$ dependence. In the evaluation of the Fourier transform and also the sum rule, this will require a regulator. Moreover, we note that by dimensional grounds the four-dimensional Fourier transform of $\Psi_{\text {pert }}$ into momentum space scales as $Q^{4}$. At first sight, this would seem to imply that the perturbative contribution to the induced Newton constant vanishes, as that contribution is given by the $Q^{2}$ term in the momentum-space function; see Eq. (17). However, that is not correct. When dealing with the physical correlation function, the perturbative result is valid over only part of the $x$ integration region. When treating the perturbative result in only the short-distance region, there is a nonzero contribution to the induced Newton constant.

Finally let us discuss the regime of intermediate energies. This can be investigated by means of the Wilson operator product expansion of time-ordered products. For the powersuppressed terms in the operator product expansion we borrow from the work that has been performed in sum-rule studies of the correlation function $\Psi$ in momentum space. The momentum-space correlator of Novikov et al. [6] is simply related to the Adler-Zee (AZ) one via

$$
\Pi_{\mathrm{AZ}}\left(q^{2}\right)=\frac{b_{0}^{2}}{64 \pi^{2}} \Pi_{\mathrm{NSVZ}}\left(q^{2}\right) .
$$

The work on the operator product expansion of $\Pi_{\mathrm{NSVZ}}\left(q^{2}\right)$ in pure QCD $\left(N_{c}=3\right)$ is described by $[8,15]$

$$
\begin{aligned}
\Pi_{\mathrm{NSVZ}}\left(q^{2}\right)= & {\left[a_{0}+a_{1} \ln \left(\frac{-q^{2}}{\mu^{2}}\right)\right]\left(-q^{2}\right)^{2} \ln \left(\frac{-q^{2}}{\mu^{2}}\right) } \\
& +\left[r_{0}+r_{1} \ln \left(\frac{-q^{2}}{\mu^{2}}\right)\right]\left\langle\alpha_{s} F^{2}\right\rangle \\
& +\left[c_{0}+c_{1} \ln \left(\frac{-q^{2}}{\mu^{2}}\right)\right] \frac{\left\langle g F^{3}\right\rangle}{\left(-q^{2}\right)} \\
& +\frac{d_{0}}{\left(-q^{2}\right)^{2}}\left\langle\alpha_{s}^{2} F^{4}\right\rangle
\end{aligned}
$$

where $\langle(\cdots)\rangle$ are gluon condensate terms:

$$
\begin{aligned}
\left\langle\alpha_{s} F^{2}\right\rangle & =\left\langle\alpha_{s} F_{\mu \nu}^{a} F^{a \mu \nu}\right\rangle, \\
\left\langle g F^{3}\right\rangle & =\left\langle g f^{a b c} F_{\mu \nu}^{a} F_{\rho}^{b \nu} F^{c \rho \mu}\right\rangle, \\
\left\langle\alpha_{s}^{2} F^{4}\right\rangle & =14\left\langle\left(\alpha_{s} f^{a b c} F_{\mu \rho}^{a} F_{\nu}^{b \rho}\right)^{2}\right\rangle-\left\langle\left(\alpha_{s} f^{a b c} F_{\mu \nu}^{a} F_{\rho \lambda}^{b}\right)^{2}\right\rangle
\end{aligned}
$$

and the various parameters appearing in Eq. (23) are given in terms of the $\alpha_{s}=g^{2} / 4 \pi$

$$
\begin{aligned}
& a_{0}=-2\left(\frac{\alpha_{s}}{\pi}\right)^{2}\left(1+\frac{51}{4} \frac{\alpha_{s}}{\pi}\right), \quad r_{0}=4 \alpha_{s}\left(1+\frac{49}{12} \frac{\alpha_{s}}{\pi}\right), \\
& c_{0}=8 \alpha_{s}^{2}, \quad d_{0}=8 \pi \alpha_{s} \\
& a_{1}=\frac{b_{0}}{2}\left(\frac{\alpha_{s}}{\pi}\right)^{3}, \quad r_{1}=-b_{0} \frac{\alpha_{s}^{2}}{\pi}, \quad c_{1}=-58 \alpha_{s}^{3} .
\end{aligned}
$$

The first (leading) term is the perturbative contribution. It can be improved by using the renormalization group and asymptotic freedom, which allows a partial resummation of the power series of logarithms appearing in it [4]. This will be briefly discussed below. In addition, the position-space forms of the various terms are defined by the Fourier transform of momentum-space results.

\section{EVALUATION}

As above we begin our considerations with the longdistance physics. By inserting Eq. (19) ito the expression for $I_{\text {IR }}$ given in the third line of Eq. (18) and performing the associated integrals, one finds

$$
I_{\mathrm{IR}}=\frac{4 \lambda^{2}}{\pi^{2} M_{g}^{4}} G_{1,3}^{3,0}\left(\frac{M_{g}^{2} t_{0}}{4} \mid \begin{array}{c}
1 \\
0,2,3
\end{array}\right),
$$

where

$$
G_{p, q}^{m, n}\left(\left.z\right|_{b_{1}, \ldots, b_{m}, \ldots, b_{q}} ^{a_{1}, \ldots, a_{n}, \ldots, a_{p}}\right)
$$

is the Meijer $G$ function [16].

For the UV contribution, we again start our considerations with the perturbative part, which we call $I_{\mathrm{UV}}^{L}$. For this we need to regularize the position-space integral. We use two methods, which lead to the same result. The QCD scale parameter is given by (at one-loop order)

$$
\begin{aligned}
\Lambda_{\mathrm{QCD}}(g(\mu), \mu) & =\mu e^{-1 /\left[b g^{2}\left(\mu^{2}\right)\right]}, \\
b & =\frac{1}{8 \pi^{2}}\left(\frac{11}{3} N_{c}-\frac{2}{3} N_{f}\right)
\end{aligned}
$$

where $\mu^{2}$ is an arbitrary subtraction point, $N_{f}=0$ and $N_{c}=3$ for gluonic QCD. In this way one gets, with another change of variables $u=\Lambda_{\mathrm{QCD}}^{2} t$ 


$$
\begin{aligned}
I_{\mathrm{UV}}^{L} & =C_{\Psi} \Lambda_{\mathrm{QCD}}^{2} \int_{0}^{u_{0}} \frac{d u}{u^{2}} \frac{\Theta(u)}{(\ln u)^{2}}, \\
\Theta(u) & =1+\sum_{n=1}^{\infty} \sum_{m=0}^{n} a_{m n} \frac{\left[\ln \left(\ln u^{-1}\right)\right]^{m}}{\left(\ln u^{-1}\right)^{n}} .
\end{aligned}
$$

The coefficients $a_{m n}$ are loop corrections of higher order. In addition we employ the restriction that $u_{0}=\Lambda_{\mathrm{QCD}}^{2} t_{0}<1$ so that the logarithm $\ln u$ does not vanish in the integration range of $I_{\mathrm{UV}}^{L}$.

Let us focus on the leading contribution. We perform the integration in two different ways. First, let us rewrite $I_{\mathrm{UV}}^{L}$ as

$$
I_{\mathrm{UV}}^{L}=C_{\Psi} \Lambda_{\mathrm{QCD}}^{2} \int_{\ln u_{0}^{-1}}^{\infty} d u e^{v} \frac{\Theta\left(e^{-v}\right)}{(v)^{2}} .
$$

In the leading order, $\Theta\left(e^{-v}\right)=1$. By introducing a regulator $e^{-\epsilon v}$ the integral can be easily done to give

$I_{\mathrm{UV}}^{L}=C_{\Psi} \Lambda_{\mathrm{QCD}}^{2}\left\{\frac{e^{x_{0}-x_{0} \epsilon}}{x_{0}}-(\epsilon-1) \Gamma\left(0, x_{0}(\epsilon-1)\right)\right\}$,

where $x_{0}=\ln u_{0}^{-1}$ and $\Gamma(a, z)$ is the incomplete gamma function. Taking the limit $\epsilon \rightarrow 0^{+}$, one gets

$$
\begin{aligned}
I_{\mathrm{UV}}^{L}= & \frac{C_{\Psi} \Lambda_{\mathrm{QCD}}^{2}}{x_{0}}\left\{e^{x_{0}}-x_{0}\left[\operatorname{Chi}\left(x_{0}\right)+\operatorname{Shi}\left(x_{0}\right)\right.\right. \\
& \left.\left.+\ln \left(-x_{0}\right)-\ln \left(x_{0}\right)\right]\right\}
\end{aligned}
$$

where $\operatorname{Chi}(z)[\operatorname{Shi}(z)]$ is the hyperbolic cosine (sine) integral. By choosing the principal branch of the logarithm, one has that $\ln \left(-x_{0}\right)-\ln \left(x_{0}\right)$ is a purely imaginary number. Hence taking the real part of $I_{\mathrm{UV}}^{L}$ leads us to

$$
I_{\mathrm{UV}}^{L}=\frac{C_{\Psi} \Lambda_{\mathrm{QCD}}^{2}}{x_{0}}\left\{e^{x_{0}}-x_{0}\left[\operatorname{Chi}\left(x_{0}\right)+\operatorname{Shi}\left(x_{0}\right)\right]\right\} .
$$

We observe that $I_{\mathrm{UV}}^{L}$ may change sign depending on the values assigned for $x_{0}$.

Now let us calculate $I_{\mathrm{UV}}^{L}$ by another method. We follow closely the discussion in Ref. [4]. First let us consider our calculations in a two-dimensional space, which yields

$$
I_{\mathrm{UV}}^{L}=C_{\Psi} \Lambda_{\mathrm{QCD}}^{2} \int_{\ln u_{0}^{-1}}^{\infty} d u e^{(d-1) v} \frac{\Theta\left(e^{-v}\right)}{(v)^{2}}
$$

with the contour of integration running along the positive real axis. Let us consider $d$ as a complex parameter; then one is interested in analytically continuing the integral to $d=2$. When $\operatorname{Re}[d]<1$ and $\operatorname{Im}[d]>0$, the integration contour can be deformed to the contour $C$ depicted in Ref. [4]. On the other hand, when $\operatorname{Re}[d]<1$ and $\operatorname{Im}[d]<0$ the contour could be deformed to a contour obtained by reflecting $C$ in the real axis. In this way, the above integral converges for any value of $\operatorname{Re}[d]$ and one can analytically continue $\operatorname{Re}[d] \rightarrow 2$. The regularization prescription must be real, as required by Hermiticity of a quantum field theory; this implies that the limit $d \rightarrow 2$ can be prescribed as the average of dimensional continuations to $d=2 \pm i \epsilon$, with $\epsilon \rightarrow 0^{+}$. That means one should take the real part of the evaluation on the contour $C$ alone at the end of the calculations. The inequivalence of the evaluations on both contours is connected to the fact that the analytic continuation of the integral to $\operatorname{Re}[d]>1$ has a branch cut running along the positive real axis from $d=1$ to infinity.

With the aforementioned prescriptions, let us perform the integral in $I_{\mathrm{UV}}^{L}$ at the leading order. We split the contour into two parts $C=C_{1} \cup C_{2}$, with the parametric representations

$$
\begin{array}{cc}
z_{1}=r e^{i \theta}, & 0<\theta<\frac{\pi}{2}, \quad r=x_{0}, \\
z_{2}=r e^{i \theta}, & \theta=\frac{\pi}{2},
\end{array}
$$

For the first contour $C_{1}$ one obtains

$$
\begin{aligned}
I_{\mathrm{UV} C_{1}}^{L}= & i \frac{C_{\Psi} \Lambda_{\mathrm{QCD}}^{2}}{x_{0}}\left\{-i x_{0}(d-1)\left(-\operatorname{Ei}\left(x_{0}(d-1)\right)\right.\right. \\
& \left.\left.+\operatorname{Ei}\left(i x_{0}(d-1)\right)\right)+e^{i x_{0}(d-1)}-i e^{x_{0}(d-1)}\right\},
\end{aligned}
$$

where $\operatorname{Ei}(z)$ is the exponential integral function. In turn, for the second contour $C_{2}$ one gets

$$
\begin{aligned}
I_{\mathrm{UV} C_{2}}^{L}= & -i \frac{C_{\Psi} \Lambda_{\mathrm{QCD}}^{2}}{x_{0}}\left\{e^{i x_{0}(d-1)}\right. \\
& \left.+i x_{0}(d-1) \Gamma\left(0,-i x_{0}(d-1)\right)\right\} .
\end{aligned}
$$

Hence $I_{\mathrm{UV}}^{L}=I_{\mathrm{UV} C_{1}}^{L}+I_{\mathrm{UV} C_{2}}^{L}$. Following the above discussed prescription, one arrives at the same result, namely Eq. (32). Incidentally, as remarked above, within dimensional regularization this leading contribution vanishes when one performs the integral for all values of $t$. Hence our result should be zero for $t_{0} \rightarrow \infty$, or $x_{0} \rightarrow-\infty$. This is precisely what happens when one takes the limit $x_{0} \rightarrow-\infty$ of Eq. (31).

Now let us calculate the contribution of the nonperturbative terms coming from the operator product expansion of $\Psi\left(x^{2}\right)$, which are the second and the third terms of Eq. (23). Using the Fourier transform to identify the position-space correlation function, one finds

$$
\begin{aligned}
\Psi_{\mathrm{UV}}^{\mathrm{OPE}}\left(x^{2}\right)= & \frac{b_{0}^{2}}{256 \pi^{4}}\left\{-\frac{4 r_{1}}{\left(x^{2}\right)^{2}}\left\langle\alpha_{s} F^{2}\right\rangle\right. \\
& \left.+\frac{\left\langle g F^{3}\right\rangle}{x^{2}}\left[c_{0}-c_{1}\left(\ln \left(\frac{x^{2} \mu^{2}}{4}\right)+2 \gamma\right)\right]\right\},
\end{aligned}
$$


where $\gamma=0.5772$ is the Euler-Mascheroni constant. Hence

$$
\begin{aligned}
I_{\mathrm{UV}}^{\mathrm{OPE}}= & \frac{b_{0}^{2} \alpha_{s}^{2}}{256 \pi^{4}}\left\{\frac{4 b_{0} t_{0}}{\pi}\left\langle\alpha_{s} F^{2}\right\rangle+\frac{t_{0}^{2}}{2}\left[4\left(2+29 \gamma \alpha_{s}\right)\right.\right. \\
& \left.\left.+29 \alpha_{s}\left(\ln \left(\frac{\mu^{4} t_{0}^{2}}{16}\right)-1\right)\right]\left\langle g F^{3}\right\rangle\right\} .
\end{aligned}
$$

Finally, collecting our results and recalling that $x_{0}=$ $\ln u_{0}^{-1}=-\ln \left(\Lambda_{\mathrm{QCD}}^{2} t_{0}\right)$, one has that

$$
\begin{aligned}
\frac{1}{16 \pi G_{\text {ind }}}= & -\frac{\pi^{2}}{96}\left\{\frac{4 \lambda^{2}}{\pi^{2} M_{g}^{4}} G_{1,3}^{3,0}\left(\frac{\bar{M}_{g}^{2} e^{-x_{0}}}{4} \mid \begin{array}{c}
1 \\
0,2,3
\end{array}\right)\right. \\
& +\frac{C_{\Psi} \Lambda_{\mathrm{QCD}}^{2}}{x_{0}}\left[e^{x_{0}}-x_{0}\left(\operatorname{Chi}\left(x_{0}\right)+\operatorname{Shi}\left(x_{0}\right)\right)\right] \\
& +\frac{b_{0}^{2} \alpha_{s}^{2}}{256 \pi^{4}}\left[\frac{4 b_{0} e^{-x_{0}}}{\Lambda_{\mathrm{QCD}}^{2} \pi}\left\langle\alpha_{s} F^{2}\right\rangle\right. \\
& +\frac{e^{-2 x_{0}}}{2 \Lambda_{\mathrm{QCD}}^{4}}\left(4\left(2+29 \gamma \alpha_{s}\right)+29 \alpha_{s}\left(\ln \left(\frac{\mu^{4} e^{-2 x_{0}}}{16 \Lambda_{\mathrm{QCD}}^{4}}\right)\right.\right. \\
& \left.\left.-1))\left\langle g F^{3}\right\rangle\right]\right\},
\end{aligned}
$$

where $\bar{M}_{g}=M_{g} / \Lambda_{\mathrm{QCD}}$.

Let us now perform a numerical analysis of the result (39). In evaluating the sum rule, we use the lattice data given in Ref. [9]. The scalar glueball mass found there is

$$
M_{g}=1.71 \pm 0.05 \pm 0.08 \mathrm{GeV},
$$

and the glueball coupling is

$$
\lambda=1.1 \pm 0.22 \mathrm{GeV}^{3} \text {. }
$$

The mass is consistent with many previous investigations. The glueball coupling turns out to be almost 4 times larger than found in a previous related study [17]. Because the glueball contribution to the induced Newton constant is negative, using a smaller coupling would make the final answer more positive. However, we see no reason not to use the most recent value as the study of Ref. [9] is a significant advance over previous work. On the other hand, for the OPE coefficients we employ the following given values of the parameters $[9,15]$ :

$$
\begin{aligned}
\left\langle\alpha_{s} F^{2}\right\rangle & =0.04 \mathrm{GeV}^{4}, \\
\left\langle g F^{3}\right\rangle & =-1.5\left\langle\alpha_{s} F^{2}\right\rangle^{3 / 2}, \\
\mu & =2 \mathrm{GeV}, \\
\alpha_{s} & =0.2 .
\end{aligned}
$$

Figure 1 illustrates the induced Newton constant as a function of $X_{0}=\sqrt{t_{0}}$. The distance interval shown in

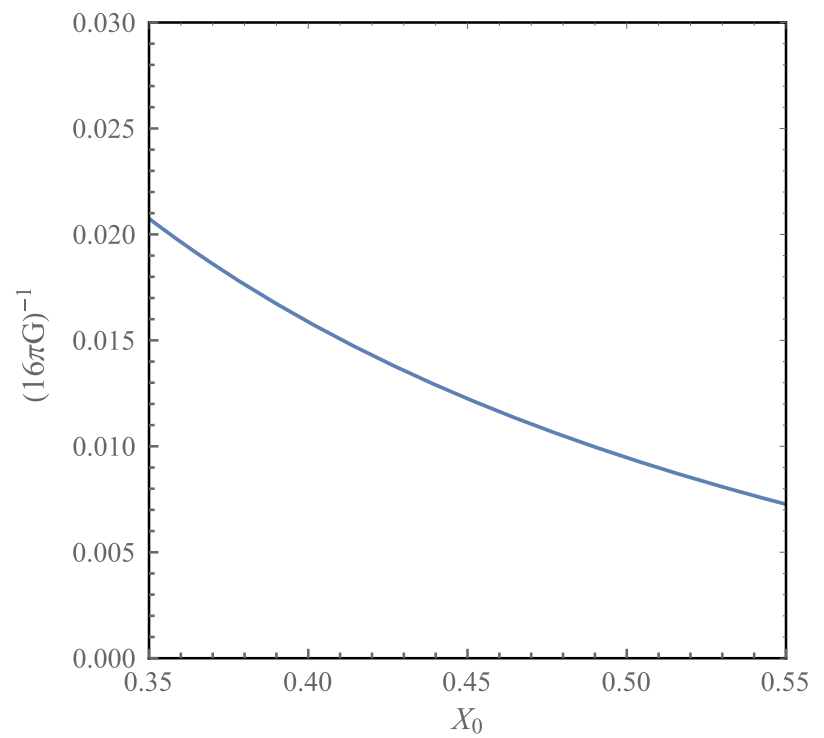

FIG. 1. $G_{\text {ind }}^{-1}$ as a function of the matching scale $X_{0}$. The values of $X_{0}$ are in units of $\mathrm{GeV}^{-1}$, and the vertical axis is in units of $\mathrm{GeV}^{2}$.

the plot corresponds to the range $X_{0}^{-1}=1.8 \mathrm{GeV}$ on the right-hand side to $X_{0}^{-1}=2.8 \mathrm{GeV}$ on the left. Because the lattice calculation utilizes a scale of $2 \mathrm{GeV}$, and reveals a glueball of $1.7 \mathrm{GeV}$ but does not investigate higher states, we quote our result for a matching scale of $X_{0}^{-1}=2 \mathrm{GeV}$ :

$$
\frac{1}{16 \pi G_{\text {ind }}}=0.0095 \pm 0.0030 \mathrm{GeV}^{2}
$$

Our error bar is determined by examining changes in the input parameters, with the most sensitive being the glueball coupling $\lambda$ of Eq. (41).

As Fig. 1 clearly reveals, $G$ is not absolutely constant in the energy range considered, which suggests that there exists some residual scale dependence in our evaluation. The matching at the scale $X_{0}$ is not perfect at the energies which we are working. This could be explained by the fact that the lattice data only reveals one glueball mass and coupling. When matching at $X_{0}^{-1} \sim 2 \mathrm{GeV}$, this should capture the bulk of the long-distance effect as the glueball mass is about $1.7 \mathrm{GeV}$. However, when the matching takes place at higher energy, the presence of extra glueball excited states probably would be relevant. Because the glueball contribution is negative, this would have the effect of decreasing the result at short distances, going in the direction of making the matching more independent of the scale. We do not have a numerical evaluation of this physics, but at least the direction of the effect is correct.

\section{DISCUSSION}

The evaluation of the Adler-Zee sum rule is an exercise in the study of QCD, but one which may have some 
implications for gravitational theories. The magnitude of the resultant shift in the effective Planck mass is typical of the QCD scale (of course) and positive. The expected scalings of the various ingredients are such that the result would stay positive for $S U(N)$ theories with larger values of $N$.

An early previous evaluation [18] also yielded a positive value of the induced Planck mass. This evaluation subtracted off the perturbative result, defined a finite energy sum rule for the remainder, and modeled the spectrum. Our result uses lattice data rather than models and more properly includes the perturbative contribution.

In addition to the induced contribution to the Planck mass, we expect a contribution to the cosmological constant, given in Eq. (1). The standard value for the gluon condensate yields a value of

$$
\Lambda_{\text {ind }}=-0.0034 \mathrm{GeV}^{4}
$$

for two light quark flavors. However, the value of the gluon condensate is not firmly known. Indeed, Holdom has given arguments that the gluon condensate could vanish, drawing attention to the absence of both experimental and theoretical evidence for a nonvanishing gluon condensate in massless QCD [19]. Some of the difficulty in direct lattice calculations is the presence of a dimensionful cutoff for the lattice, and also disentangling the gluonic contribution from that of massive quarks. If this assertion is correct, it would have important implications for the use of induced effects in gravity theories. Our induced shift in the Planck mass remains positive and within the quoted error bars if we set the gluon condensate to zero.

It is evident from the derivation that the shift in the value of $G$ due to QCD is only valid for wavelengths greater than the QCD scale. For shorter wavelengths the effect is different, and the effective value of $G$ above the QCD scale will be different. Moreover the effect on the graviton propagator, defined by the graviton two-point function, will be a more complicated function of the momentum, including the development of an imaginary component, bringing in the Lee-Wick mechanism [20-22]. While the case of QCD is not likely to be of phenomenological importance, because the QCD scale is so far below the Planck scale, if there are other strongly interacting gauge theories at much higher energy, there could be important consequences. Some recent suggestions include the interactions of the spin connection [12] and even of the gravitational field itself [13]. These effects deserve further study.

\section{ACKNOWLEDGMENTS}

We thank B. Holdom, J. Ren, P. Vanhove, K.-F. Liu, C. Kiefer and L. H. Ford for useful discussions. This work has been supported in part by the National Science Foundation under Grant No. NSF PHY15-20292.

\section{APPENDIX: THE COSMOLOGICAL CONSTANT SUM RULE}

In this appendix we elucidate the mismatch involving a two-point representation for the cosmological constant, where the works of NSVZ [6] and of Brown and Zee [14] yield sum rules that differ by a factor of 2 . For QCD, the correct one is that of NSVZ, which reads

$$
\Lambda_{\text {ind }}=-\frac{i}{16} \int d^{4} z\langle T\{\bar{T}(z) \bar{T}(0)\}\rangle=-\frac{b_{0}}{32}\left\langle\frac{\alpha_{s}}{\pi} F_{\mu \nu}^{a} F^{a \mu \nu}\right\rangle
$$

where

$$
b_{0}=\frac{11}{3} N_{c}-\frac{2}{3} N_{f}
$$

Here $N_{f}$ is the number of quark species in the theory. For purely gluonic QCD one has $N_{f}=0$ and $N_{c}=3$.

The equality on the right side is the sum-rule identity given by NSVZ, and we have used Eq. (1) to relate that result to $\Lambda_{\text {ind }}$ in order to obtain the equality on the left side. The sum rule of Brown and Zee corresponds to the lefthand equality but with a coefficient that is twice as large, with the $i / 16$ being replaced by $i / 8$.

The issue hinges on the two-graviton coupling called $\tau_{\mu \nu, \alpha \beta}(x)$ in Eq. (7). When Brown and Zee expanded the action they included only the linear coupling $h^{\mu \nu} T_{\mu \nu}$. By matching the resultant Lagrangian to that of a cosmological constant, their sum rule was obtained. We can see how this is changed by including the two-graviton coupling. First let us exhibit the representations for $\Lambda_{\text {ind }}$. Recalling that $h_{\mu \nu}=(1 / 4) \eta_{\mu \nu} h$, one has that $\sqrt{-g}=$ $1+(1 / 2) h+(1 / 16) h^{2}$. Since $\sqrt{-g} \mathcal{L}_{\text {eff }}^{(0)}\left[g_{\mu \nu}\right]=-\sqrt{-g} \Lambda_{\text {ind }}$, a comparison with Eq. (10) leads to the following representations for the cosmological constant:

$$
\begin{aligned}
& \Lambda_{\text {ind }}=\frac{1}{4}\langle T(x)\rangle, \\
& \Lambda_{\text {ind }}=-\frac{1}{4} \eta^{\mu \nu} \eta^{\rho \sigma}\left\langle\tau_{\mu \nu, \rho \sigma}(x)\right\rangle-\frac{i}{8} \int d^{4} z\langle T\{\bar{T}(z) \bar{T}(0)\}\rangle .
\end{aligned}
$$

The second of these is the correct sum rule in a generic theory. The result of Brown and Zee is obtained if one drops $\tau_{\mu \nu, \rho \sigma}$. However, in Yang-Mills theories, the twograviton coupling does contribute, and resolves the discrepancy in the sum rules. The Yang-Mills Lagrangian in a generic curved background is given by

$$
\sqrt{-g} \mathcal{L}_{\mathrm{YM}}=\sqrt{-g}\left[-\frac{1}{4} F_{\mu \nu}^{a} F^{a \mu \nu}\right],
$$


where the index $a$ is summed over the generators of the gauge group $G$. The field strength is given by

$$
F_{\mu \nu}^{a}=\partial_{\mu} A_{\nu}^{a}-\partial_{\nu} A_{\mu}^{a}+g f^{a b c} A_{\mu}^{b} A_{\nu}^{c}
$$

where $f^{a b c}$ are the structure constants of $G$. For weak fields the metric can be expanded using $g_{\mu \nu}=\eta_{\mu \nu}+h_{\mu \nu}$. One finds that

$$
\begin{aligned}
\sqrt{-g} \mathcal{L}_{\mathrm{YM}}= & -\frac{1}{4} F_{\mu \nu}^{a} F^{a \mu \nu}-\frac{1}{2} h^{\mu \nu} T_{\mu \nu} \\
& +\frac{1}{4} h^{\mu \nu} h^{\alpha \beta} \tau_{\mu \nu, \alpha \beta}+\mathcal{O}\left(h^{3}\right)
\end{aligned}
$$

where the energy-momentum tensor for the Yang-Mills field in Minkowski space-time is given by

$$
T_{\mu \nu}=-F_{\lambda \mu}^{a} F_{\nu}^{a \lambda}+\frac{1}{4} \eta_{\mu \nu} F_{\alpha \beta}^{a} F^{a \alpha \beta} .
$$

In addition, the tensor $\tau_{\mu \nu, \alpha \beta}$ reads

$$
\begin{aligned}
\tau_{\mu \nu, \alpha \beta}= & -F_{\mu \alpha}^{a} F_{\nu \beta}^{a}+\frac{1}{4} \mathcal{P}_{\alpha \beta \mu \nu} F_{\gamma \delta}^{a} F^{a \gamma \delta} \\
& -2 \eta_{\alpha \nu} F_{\lambda \mu}^{a} F_{\beta}^{a \lambda}+\eta_{\alpha \beta} F_{\kappa \mu}^{a} F_{\nu}^{a \kappa}
\end{aligned}
$$

where

$$
\mathcal{P}_{\alpha \beta \mu \nu}=\frac{1}{2}\left(\eta_{\alpha \mu} \eta_{\beta \nu}+\eta_{\alpha \nu} \eta_{\beta \mu}-\eta_{\alpha \beta} \eta_{\mu \nu}\right) .
$$

Now let us prove the result (A1). In order to calculate $\Lambda_{\text {ind }}$, one needs an expression for $T(x)$. With the introduction of a dynamical scale-invariance breaking, one gets $T(x) \neq 0$. This is the well-known trace anomaly. The trace anomaly formula for pure QCD is given by (see Ref. [4] and references cited therein)

$$
T(x)=\frac{\beta(g)}{2 g} F_{\mu \nu}^{a} F^{a \mu \nu},
$$

where $\beta(g)$ is the renormalization-group beta function, which in the lowest order is given by

$$
\beta(g)=-\frac{1}{2} b g^{3} .
$$

In this way one also finds

$$
\eta^{\mu \nu} \eta^{\rho \sigma} \tau_{\mu \nu \rho \sigma}=\frac{\beta(g)}{2 g} F_{\gamma \delta}^{a} F^{a \gamma \delta}=T(x) .
$$

In Eqs. (A9) and (A11) it is to be understood that $F_{\mu \nu}^{a}$ is the renormalized field strength. From the first of the relations presented in Eq. (A2), one gets

$$
\Lambda_{\text {ind }}=-\frac{b_{0}}{32}\left\langle\frac{\alpha_{s}}{\pi} F_{\mu \nu}^{a} F^{a \mu \nu}\right\rangle .
$$

Now let us discuss the two-point representation for the induced cosmological constant. Using the sum rule derived in Ref. [6], which states that

$$
i \int d z\langle T\{\bar{T}(z) \bar{T}(0)\}\rangle=\frac{b_{0}}{2}\left\langle\frac{\alpha_{s}}{\pi} F_{\mu \nu}^{a} F^{a \mu \nu}\right\rangle
$$

it is easy to see that the second of the relations presented in Eq. (A2) produces the same result for $\Lambda_{\text {ind. }}$. This proves our assertion.

We do not evaluate the cosmological constant sum rule numerically because of the possibility of delta function OPE contributions to the position-space sum rule [7]. Because of the extra powers of $x^{2}$, these do not influence the Adler-Zee formula, but they would enter into the cosmological constant sum rule. For the cosmological constant, Eq. (1) is still the most reliable estimate.
[1] S. L. Adler, A formula for the induced gravitational constant, Phys. Lett. B 95, 241 (1980).

[2] A. Zee, Spontaneously generated gravity, Phys. Rev. D 23, 858 (1981).

[3] A. Zee, Calculation of Newton's gravitational constant in infrared-stable Yang-Mills theories, Phys. Rev. Lett. 48, 295 (1982).

[4] S. L. Adler, Einstein gravity as a symmetry breaking effect in quantum field theory, Rev. Mod. Phys. 54, 729 (1982); Erratum, Rev. Mod. Phys. 55, 837(E) (1983).
[5] A. D. Sakharov, Vacuum quantum fluctuations in curved space and the theory of gravitation, Dokl. Akad. Nauk Ser. Fiz. 177, 70 (1967) [Sov. Phys. Dokl. 12, 1040 (1968)] [Sov. Phys. Usp. 34, 394 (1991)] [Gen. Relativ. Gravit. 32, 365 (2000)].

[6] V. A. Novikov, M. A. Shifman, A. I. Vainshtein, and V. I. Zakharov, In a search for scalar gluonium, Nucl. Phys. B165, 67 (1980).

[7] V. A. Novikov, M. A. Shifman, A. I. Vainshtein, and V. I. Zakharov, Calculations in external fields in quantum 
chromodynamics. Technical review, Fortschr. Phys. 32, 585 (1984).

[8] E. Bagan and T. G. Steele, Mass of the scalar glueball: Higher loop effects in the QCD sum rules, Phys. Lett. B 243, 413 (1990).

[9] Y. Chen et al., Glueball spectrum and matrix elements on anisotropic lattices, Phys. Rev. D 73, 014516 (2006).

[10] K. S. Stelle, Renormalization of higher derivative quantum gravity, Phys. Rev. D 16, 953 (1977).

[11] E. S. Fradkin and A. A. Tseytlin, Renormalizable asymptotically free quantum theory of gravity, Phys. Lett. B 104, 377 (1981); J. Julve and M. Tonin, Quantum gravity with higher derivative terms, Nuovo Cimento Soc. Ital. Fis. 46B, 137 (1978).

[12] J. F. Donoghue, Is the spin connection confined or condensed?, Phys. Rev. D 96, 044003 (2017); J. F. Donoghue, Conformal model of gravitons, Phys. Rev. D 96, 044006 (2017); Quartic propagators, negative norms and the physical spectrum, Phys. Rev. D 96, 044007 (2017).

[13] A. Zee, Einstein gravity emerging from quantum Weyl gravity, Ann. Phys. (N.Y.) 151 (1983) 431; B. Holdom and J. Ren, QCD analogy for quantum gravity, Phys. Rev. D 93, 124030 (2016); Quadratic gravity: From weak to strong, Int. J. Mod. Phys. D 25, 1643004 (2016); A. Salvio and A. Strumia, Agravity, J. High Energy Phys. 06 (2014) 080; M. B. Einhorn and D. R. T. Jones, Naturalness and dimensional transmutation in classically scale-invariant gravity, J. High Energy Phys. 03 (2015) 047; T. Jones and M. Einhorn, Quantum gravity and dimensional transmutation, Proc. Sci., PLANCK2015 (2015) 061; G. Narain and R. Anishetty, Short distance freedom of quantum gravity, Phys. Lett. B 711, 128 (2012); A. V. Smilga, Ghost-free higher-derivative theory, Phys. Lett. B 632, 433 (2006); A. V. Smilga, Spontaneous generation of the Newton constant in the renormalizable gravity theory, in Proceedings of the Conference on Group Theoretical Methods in Physics, Zvenigorod, USSR, 1982, Vol. 2, edited by M. A. Markov, V. I. Manko, and A. E. Shabad (Nauka, Moscow, 1983), p. 73; E. Tomboulis, Renormalizability and asymptotic freedom in quantum gravity, Phys. Lett. B 97, 77 (1980);
S. D. Odintsov and I. L. Shapiro, General relativity as the low-energy limit in higher derivative quantum gravity, Classical Quantum Gravity 9, 873 (1992) [Teor. Mat. Fiz. 90, 469 (1992)] [Theor. Math. Phys. 90, 319 (1992)]; K. Akama, Y. Chikashige, T. Matsuki, and H. Terazawa, Gravity and electromagnetism as collective phenomena: A derivation of Einstein's general relativity, Prog. Theor. Phys. 60, 868 (1978).

[14] L. S. Brown and A. Zee, Response to gravitational probes and induced Newton's constant, J. Math. Phys. (N.Y.) 24, 1821 (1983).

[15] H. Forkel, Scalar gluonium and instantons, Phys. Rev. D 64, 034015 (2001).

[16] A. P. Prudnikov, Y. A. Brychkov, and O. I. Marichev, Integrals and Series, Vol. 2 (Gordon and Breach, London, 1986).

[17] Y. Liang, K. F. Liu, B. A. Li, S. J. Dong, and K. Ishikawa, Lattice calculation of glueball matrix elements, Phys. Lett. B 307, 375 (1993).

[18] N. V. Krasnikov and A. A. Pivovarov, The use of finite energy sum rules for the calculation of the induced gravitational constant, Phys. Lett. B 161, 373 (1985).

[19] B. Holdom, Does massless QCD have vacuum energy?, New J. Phys. 10, 053040 (2008); Mass gap without vacuum energy, Phys. Lett. B 681, 287 (2009).

[20] T. D. Lee and G. C. Wick, Negative metric and the unitarity of the $S$ matrix, Nucl. Phys. B9, 209 (1969); Unitarity in the $\mathrm{N} \theta \theta$ sector of soluble model with indefinite metric, Nucl. Phys. B10, 1 (1969); Finite theory of quantum electrodynamics, Phys. Rev. D 2, 1033 (1970); R. E. Cutkosky, P. V. Landshoff, D. I. Olive, and J.C. Polkinghorne, A nonanalytic $S$ matrix, Nucl. Phys. B12, 281 (1969).

[21] E. Tomboulis, $1 / N$ expansion and renormalization in quantum gravity, Phys. Lett. B 70, 361 (1977); I. Antoniadis and E. T. Tomboulis, Gauge invariance and unitarity in higher derivative quantum gravity, Phys. Rev. D 33, 2756 (1986).

[22] B. Grinstein, D. O'Connell, and M. B. Wise, Causality as an emergent macroscopic phenomenon: The Lee-Wick $O(N)$ model, Phys. Rev. D 79, 105019 (2009); The Lee-Wick standard model, Phys. Rev. D 77, 025012 (2008). 